\title{
Benthic macroinvertebrates as bioindicators of water quality in an Atlantic forest fragment
}

\author{
Augusto Oliveira \& Marcos Callisto
}

Laboratório de Ecologia de Bentos, Departamento de Biologia Geral, Instituto de Ciências Biológicas, Universidade Federal de Minas Gerais, Caixa Postal 486, 31270-901 Belo Horizonte, MG, Brazil. (augusto@limnologica.com.br; callistom@ufmg.br)

\begin{abstract}
The objective of this study was to evaluate benthic macroinvertebrate communities as bioindicators of water quality in five streams located in the "Reserva Particular do Patrimônio Natural" (RPPN) Mata Samuel de Paula and its surroundings, in the municipality of Nova Lima near the city of Belo Horizonte, Minas Gerais State, southeastern Brazil. This region has been strongly modified by human activities including mining and urbanization. Samples were collected in the field every three months between August 2004 and November 2005 , totaling six samplings in the rainy and dry seasons. This assessment identified one area ecologically altered while the other sampling sites were found to be minimally disturbed systems, with well-preserved ecological conditions. However, according to the Biological Monitoring Work Party (BMWP) and the Average Score Per Taxon (ASPT) indices, all sampling sites had excellent water quality. A total of 14,952 organisms was collected, belonging to 155 taxa (148 Insecta, two Annelida, one Bivalvia, one Decapoda, one Planariidae, one Hydracarina, and one Entognatha). The most abundant benthic groups were Chironomidae (47.9\%), Simuliidae (12.3\%), Bivalvia (7.5\%), Decapoda (6.1\%), Oligochaeta (5.2\%), Polycentropodidae (3.7\%), Hydropsychidae (2.5\%), Calamoceratidae (1.8\%), Ceratopogonidae (1.7\%), and Libellulidae (1.2\%). The assessment of the benthic functional feeding groups showed that $34 \%$ of the macroinvertebrates were collector-gatherers, $29 \%$ predators, $24 \%$ collector-filterers, $8 \%$ shredders, and 5\% scrapers. The RPPN Mata Samuel de Paula comprises diversified freshwater habitats that are of great importance for the conservation of many benthic taxa that are intolerant to organic pollution.
\end{abstract}

KEYWORDS. Benthic inventory, biodiversity, seasonal distribution, protected areas, functional feeding groups.

RESUMO. Macroinvertebrados bentônicos como bioindicadores de qualidade de água em um fragmento de Mata Atlântica. $\mathrm{O}$ objetivo deste estudo foi avaliar as comunidades de macroinvertebrados bentônicos como bioindicadoras de qualidade de água em cinco riachos localizados na Reserva Particular do Patrimônio Natural (RPPN) Mata Samuel de Paula, no município de Nova Lima, próximo à cidade de Belo Horizonte, estado de Minas Gerais, sudeste do Brasil. A região tem sido severamente alterada por atividades antrópicas incluindo mineração e urbanização. As amostras foram coletadas em campo trimestralmente entre agosto de 2004 e novembro de 2005, totalizando 6 campanhas nas estações seca e chuvosa. Este estudo identificou um riacho como alterado ecologicamente enquanto os demais foram caracterizados como minimamente alterados, com condições ecológicas bem preservadas. No entanto, de acordo com os índices Biological Monitoring Work Party (BMWP) e Average Score Per Taxon (ASPT), todos os riachos possuem qualidade de água excelente. No total, 14.952 organismos foram coletados, pertencendo a 155 táxons (148 Insecta, 2 Annelida, 1 Bivalvia, 1 Decapoda, 1 Planariidae, 1 Hydracarina e 1 Entognatha). Os grupos bentônicos mais abundantes foram Chironomidae $(47,9 \%)$, Simuliidae $(12,3 \%)$, Bivalvia (7,5\%), Decapoda (6,1\%), Oligochaeta (5,2\%), Polycentropodidae $(3,7 \%)$, Hydropsychidae $(2,5 \%)$, Calamoceratidae $(1,8 \%)$, Ceratopogonidae $(1,7 \%)$ e Libellulidae $(1,2 \%)$. A classificação dos grupos tróficos funcionais evidenciou que $34 \%$ dos macroinvertebrados eram coletores-catadores, $29 \%$ predadores, $24 \%$ coletores-filtradores, $8 \%$ fragmentadores e 5\% raspadores. A RPPN Mata Samuel de Paula protege hábitats aquáticos diversificados de grande importância para a conservação de muitos táxons de macroinvertebrados bentônicos que são intolerants à poluição.

PALAVRAS-CHAVE. Inventários bentônicos, biodiversidade, distribuição sazonal, áreas protegidas, grupos tróficos funcionais.

The Atlantic forest is one of the more diverse Brazilian biomes, and one of the most threatened worldwide (MYers et al., 2000). The exploitation of its natural resources by humans, together with urban growth and the increase in the amount of effluents produced, have intensified its degradation process. Nowadays, a small part of the original extension of this biome is still preserved in Brazil, and is protected in small conservation units that are usually located on private properties, and termed Reserva Particular do Patrimônio Natural (RPPN).

The aquatic communities in the Atlantic forest are poorly known. River degradation can threaten the existence of many species before they are even described, because aquatic ecosystems are most affected by human activities (SAUNDERS et al., 2002).

Clearance of riparian vegetation is one of the degrading factors that affect aquatic ecosystems in the Atlantic forest. The vegetation reduces erosive processes and increases streambank stability, helping to maintain fluvial habitats, and provides litter input and shade (FrANÇA et al., 2009).
The conservation of the Brazilian freshwater ecosystems and their biodiversity depends in part on the establishment of conservation units that encompass entire hydrographic basins, and that are based on studies of their ecological and biological conditions (PAZ et al., 2008). Knowledge about the health status of aquatic ecosystems and the value of the potential services that they can provide to humans allows optimal and sustainable use of the available resources (CONSTANZA et al., 1997).

Among the communities that are considered bioindicators of water quality, the most commonly used are the benthic macroinvertebrates (BONADA et al., 2006), because they have several characteristics that make them easy to study, and show clear responses when faced with adverse environmental conditions (MORENo et al., 2009). The structure of the benthic communities in an aquatic ecosystem reflects its ecological conditions, including habitat heterogeneity (HEINO et al., 2003) and water quality (SOLDNER et al., 2004). Some abiotic factors such as temperature, $\mathrm{pH}$, electrical conductivity, dissolved 
oxygen in water column, granulometric composition and organic matter content in sediment, among others, determine the distribution of benthic macroinvertebrate communities (Allan, 1995).

Macroinvertebrates have an important role in the trophic structure of aquatic ecosystems, feeding on living or decomposing organic matter and serving as food for other invertebrates and vertebrates (Moulton et al., 2010). The proportions of different trophic groups are heavily influenced by physical and chemical conditions of aquatic ecosystems (OLIVEIRA et al., 1999). In headwater streams in the Atlantic forest where the riverbed is shaded by a closed canopy, the riparian vegetation is the main source of food for benthic macroinvertebrates, supplying organic debris that are used for shelter or food (MORETTI et al., 2009).

This study evaluated the structure and composition of benthic macroinvertebrate communities in streams of an important Atlantic forest fragment remaining in the state of Minas Gerais, Brazil. The water quality in the streams was evaluated based on a taxonomic inventory of benthic communities and measurements of physical and chemical conditions of the water column and sediments. The objective was to inventory the benthic biodiversity and assess differences on a spatial scale and between the rainy and dry periods. All parameters were evaluated in order to answer two questions: 1 . are there differences among sampling stations? 2. are there seasonal differences? This information may provide important tools for the selection of headwaters and spring areas to be the reference sites for biomonitoring programs of the upper course of the Velhas river, the main tributary of the upper São Francisco river basin.

\section{MATERIAL AND METHODS}

Study area. The "Reserva Particular do Patrimônio Natural" (RPPN) Mata Samuel de Paula (1959'51.8' S, $43^{\circ} 52^{\prime} 19.3^{\prime \prime} \mathrm{W}$ ) encompasses an important forest fragment of 147 ha in Nova Lima municipality in the metropolitan area of the city of Belo Horizonte, in the state of Minas Gerais, Brazil. Surrounded by residential areas and by the Nova Lima municipality, this RPPN suffers from diverse human pressures due to possible unauthorized settlement by squatters within its boundaries, and industrial activities and the growth of residential areas in its surroundings. Part of the boundary of the RPPN is close to the urban area of Nova Lima. Another boundary is formed by a stream called Cristais creek that meets the Cardoso creek, forming the basin of another stream, the Água Suja which is part of the upper course of the Velhas river basin. Five sampling stations were defined: three inside the RPPN (stations 1, 2, and 4) and two located in the surroundings of the conservation unit (stations 3 and 5) (Fig. 1).

Sampling and identification. Sampling was carried out in six periods during the rainy (November 2004, February and November 2005) and dry seasons (August 2004, May and August 2005). In each field period, fifteen macroinvertebrate samples were collected (three in each stream) using a $0.250 \mathrm{~mm}$ mesh Surber sampler with a sampling area of $0.09 \mathrm{~m}^{2}(30 \mathrm{~cm} \mathrm{x} 30 \mathrm{~cm})$. The macroinvertebrate communities were evaluated in 18 samples from each stream, for a total of 90 samples.

Macroinvertebrate samples were stored in plastic bags, fixed with formaldehyde (10\%), and transported to the Laboratório de Ecologia de Bentos at the Universidade Federal de Minas Gerais (UFMG) for analysis.

Samples were washed over 1.00, 0.5, and $0.25 \mathrm{~mm}$ mesh sieves, and arranged in trays for identification to the lowest taxonomic level possible. The specimens collected were deposited in the "Coleção de Referência de Macroinvertebrados Bentônicos" of the Instituto de Ciências Biológicas in the UFMG.

The specimens found in the streams were classified into five functional trophic groups (Tab. I), defined by their feeding strategy as collector-gatherers, predators, collector-filterers, shredders, and scrapers (MERRITT \& Cummins 1996; Cummins et al., 2005).

Environmental characterization. During each sampling period, a rapid evaluation protocol to assess the ecological conditions was applied (CALlisto et al., 2002) at each sampling station. This protocol evaluates 22 parameters that receive higher scores for natural conditions and lower scores for altered conditions, which makes it possible to classify the conservation status of the streams. Some ecological aspects such as land use (human influence, erosion, presence and maintenance of riparian vegetation, bankfull incision), stream features (canopy cover, sediment odor and presence of oil, substrate type and particle size, siltation, channel modifications), water characteristics (odor, oil presence, transparency, rapids and pools, discharge), and the presence of aquatic macrophytes were evaluated.

A Horiba U22 water quality meter was used in situ to estimate water temperature $\left({ }^{\circ} \mathrm{C}\right), \mathrm{pH}$, electrical conductivity $(\mu \mathrm{S} / \mathrm{cm})$, and turbidity (NTU). The dissolved oxygen concentration $(\mathrm{mg} / \mathrm{l})$ was determined by the Winkler method. Water samples were collected for laboratory analysis in order to determine total-P, total-N, $\mathrm{PO}_{4}-\mathrm{P}, \mathrm{NO}_{3}^{-}, \mathrm{NO}_{2}^{-}, \mathrm{NH}_{4}^{+}$, and $\mathrm{SiO}_{2}$ concentrations and the total alkalinity, following MACKERETH et al. (1978). The concentration of chlorophyll-a in water samples was determined following LORENZEN (1967).

Two sediment samples were collected in each sampling station in each period, to evaluate the granulometric composition and the organic-matter content, following Suguio (1973).

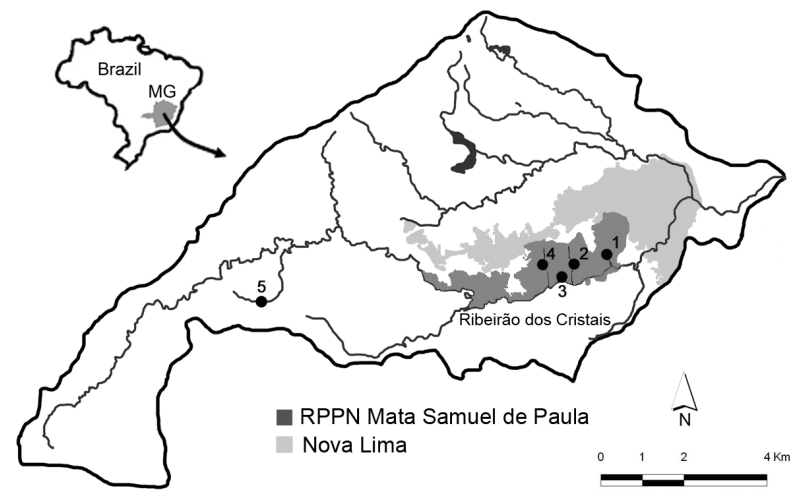

Figure 1. Location of the sampled stations (1 up to 5) at the basin of the upper Velhas river, Nova Lima, MG. 
Data analysis. The structure of the macroinvertebrate communities was evaluated using the Shannon-Wiener diversity index and Pielou's equitability index (MAGURRAN, 1991). Total organism densities (individuals $\mathrm{m}^{-2}$ ), relative abundance (\% of individuals), and taxonomic richness (total number of taxa) were estimated in each of the streams.

The BMWP (Biological Monitoring Work Party) index was applied following JUNQUEIRA et al. (2000) for the classification of water quality in the streams. The BMWP value was divided by the number of families used in its determination, in order to calculate the ASPT (Average Score Per Taxon) index (ARMitAge et al., 1983).

The granulometric composition of sediment samples was evaluated by the Shannon-Wiener diversity and Pielou evenness indices.

The structure and composition of the macroinvertebrate communities in the streams were evaluated using one-way ANOVA, and the significant differences found were verified using the Tukey test. The

Table I. Functional feeding groups of benthic macroinvertebrates sampled in the studied headwater streams at the "Reserva Particular do Patrimônio Natural" Mata Samuel de Paula, Nova Lima, MG.

\begin{tabular}{|c|c|}
\hline Shredders & Predators \\
\hline Curculionidae - larvae & Hirudinea \\
\hline Ptilodactylidae - larvae & Dytiscidae \\
\hline Gripopterygidae & Gyrinidae \\
\hline Calamoceratidae & Hydrophilidae - larvae \\
\hline Odontoceridae & Staphylinidae - adults \\
\hline Hydroptilidae - Orthotrichia sp. & Ceratopogonidae \\
\hline Decapoda & Chaoboridae \\
\hline Collector-gatherers & Empididae \\
\hline Collembola & Tabanidae \\
\hline Elmidae - larvae & Tanypodinae \\
\hline Scirtidae - larvae & Tipulidae \\
\hline Hydrophilidae - adults & Belostomatidae \\
\hline Chironominae & Gerridae \\
\hline Culicidae & Naucoridae \\
\hline Diamesinae & Nepidae \\
\hline Dixidae & Notonectidae \\
\hline Stratiomyidae & Veliidae \\
\hline Baetidae & Corydalidae \\
\hline Caenidae - Caenis sp. & Aeshnidae \\
\hline Leptohyphidae & Calopterygidae \\
\hline Leptophlebiidae - Farrodes sp. & Coenagrionidae \\
\hline Hydroptilidae - Oxyethira sp. & Gomphidae \\
\hline Leptoceridae - Nectopsyche sp. & Libellulidae \\
\hline Oligochaeta & Megapodagrionidae \\
\hline Collector-filterers & Perlidae \\
\hline Bivalvia & Leptoceridae - Oecetis sp. \\
\hline Simuliidae & Hydrobiosidae \\
\hline Hydropsychidae & Hidracarina \\
\hline Philopotamidae & Planariidae \\
\hline \multicolumn{2}{|l|}{ Polycentropodidae } \\
\hline \multicolumn{2}{|l|}{ Scrapers } \\
\hline \multicolumn{2}{|l|}{ Elmidae - adults } \\
\hline \multicolumn{2}{|l|}{ Psephenidae - larvae } \\
\hline \multicolumn{2}{|l|}{ Orthocladiinae } \\
\hline \multicolumn{2}{|l|}{ Psychodidae } \\
\hline \multicolumn{2}{|l|}{ Lepidoptera } \\
\hline \multicolumn{2}{|l|}{ Helicopsychidae } \\
\hline Hydroptilidae - Hydroptila sp.; $N$ & ichia sp. \\
\hline
\end{tabular}

variations in richness values, total density, EPT (Ephemeroptera, Plecoptera, and Trichoptera) percentage, Chironomidae and Oligochaeta percentages, and functional feeding groups were evaluated using ANOVA between seasons and streams. Benthic community parameters were used as dependent variables and the sampling periods as independent variables, totaling 18 replicates assessed for each sampling station.

Multi dependent analysis of variance (MANOVA) was used to test the difference between rainy and dry seasons of physical and chemical parameters for each sampling station. Variables of table II were the dependent variables, and the rainy and dry seasons were the independent variables (predictor factors) in all analysis.

\section{RESULTS}

Abiotic factors and ecological conditions. The assessment of ecological conditions in the sampling sites provided a better knowledge of the physical and chemical characteristics and the benthic macroinvertebrates. Based on the rapid assessment of the ecological conditions, the sampling stations were classified in: station 1 (altered) - a dam was present, riparian vegetation partially removed, stable banks, and high accumulation of allochthonous leaves and branches on the stream bottom; station 2 (minimally disturbed) - some water-supply pipes were present, trash on the banks, well-preserved riparian vegetation, slight erosion of streambanks, muddy sediment; station 3 (minimally disturbed) - moderate erosion of streambanks, high diversity of aquatic habitats, high frequency of riffles and turbulent waters, rocky bed with sand deposits; station 4 (minimally disturbed) - the riparian vegetation was well developed; high diversity of substrate types and habitats; leaf deposits, trunks, and branches in decomposition; cobbles, pebbles, and sand; presence of riffles and pools; total canopy cover; no erosion of streambanks; station 5 (minimally disturbed) - riparian vegetation $20 \mathrm{~m}$ wide, surrounded by sheds and houses, unstable banks, some evidence of erosion and siltation, high substrate richness, leaf deposits, numerous riffles and pools.

The physical and chemical parameters measured in the water at all the sampling stations are shown in table II. Significant differences between rainy and dry seasons in physical and chemical parameters were found only in station 1 (MANOVA, Wilks Lambda ${ }_{1.4}=601.38 ; \mathrm{p}=0.031$ ). The electrical conductivity was low at all the stations. The concentrations of dissolved oxygen were higher at stations 3, 4, and 5, where rapids were more frequent.

The sediment samples from the five stations had organic-matter contents that ranged from $3.0 \%$ D.W. at station 3 to $66.5 \%$ D.W. at station 1 (Tab. II). Sediments at stations 1 and 2 were dominated by very fine sand and silt+clay. At station 3, there was a great difference between the sampling periods. Station 4 had a sandy substrate, and station 5 had pebbles and cobbles as the main granulometric particles (Tab. II).

Benthic macroinvertebrates. From all five sites, a total of 14,952 specimens were collected. These belonged to 155 taxa, as follows: 148 Insecta, two Annelida, one Bivalvia, one Decapoda, one Tricladida, 
one Hydracarina, and 1 Entognatha (Tab. III). The most abundant taxa were Chironomidae $(47.9 \%)$ and Simuliidae $(12.3 \%$ ), representing $c a .60 \%$ of the total benthic macroinvertebrates.

The communities contained many rare taxa, since the largest component of taxonomic richness found in this study (90 taxa) comprised only $9.9 \%$ of the specimens collected, with several groups comprising less than $1 \%$ of the total. Despite the high number of taxa found, these communities of benthic macroinvertebrates were dominated by only two or three taxa, as observed at station 1 (Chironomidae) and station 3 (Simuliidae and Chironomidae).

The lowest density was found at station 5 in the dry $\left(610.9 \pm 596.7\right.$ ind. $\left.\mathrm{m}^{-2}\right)$ and rainy $\left(64.0 \pm 94.1\right.$ ind. $\left.\mathrm{m}^{-2}\right)$ seasons. The highest densites occurred at station 1 $\left(5,116.7 \pm 6,067.7\right.$ ind. $\mathrm{m}^{-2}$ in the dry, and 2,322.5 $\pm 3,154.7$ ind. $\mathrm{m}^{-2}$ in the rainy season).

The taxonomic richness of benthic macroinvertebrates during the dry season was lowest at station $5(14.6 \pm 8.1$ taxa), and highest at station $2(18.2 \pm 5.4$ taxa). During the wet season, the richness values were $4.0 \pm 4.5$ taxa at station 5 and $19.1 \pm 1.5$ taxa at station 4 . Significant differences in taxonomic richness among the stations were only found during the wet season (ANOVA, $\mathrm{F}_{4.44}=5.32$; $\mathrm{p}=0.0016$ ), with differences between stations 2 and 5 (Tukey's test $=0.0108)$, stations 3 and 4 (Tukey's test $=$ 0.0457 ), and stations 4 and 5 (Tukey's test $=0.0037$ ).

Based on the benthic macroinvertebrates collected in the five sampling stations, the water quality was classified as excellent, with high values of both the BMWP and ASPT indices. These streams contain sensitive benthic macroinvertebrates with low tolerance to environmental degradation and requirements for high water quality in terms of physical and chemical parameters (Tab. IV).

The taxonomic richness was significantly different between sampling stations $\left(\mathrm{F}_{4.44}=5.32 ; \mathrm{p}=0.0016\right)$ in the rainy season, and the EPT in the dry season $\left(\mathrm{F}_{4.44}=7.27\right.$; $\mathrm{p}=0.00016)($ Tab. V).
The collector-gatherers were the most abundant trophic group, comprising 34\% of the macroinvertebrates. The second most abundant were the predators, with $29 \%$ of the total. The collector-filterers comprised $24 \%$, followed by shredders $(8 \%)$ and scrapers $(5 \%)$.

The highest proportions of shredders, represented by Decapoda and Calamoceratidae, were found at station 4 during November 2004 and February and November 2005, and also at station 2. At station 5, the main shredders were Gripopterygidae (Paragripopteryx Burmeister, 1839 and Tupiperla Burmeister, 1839) and Calamoceratidae (Phylloicus Mueller, 1880). The scrapers were found in low proportions, except at station 4 during May 2005, where they were mainly represented by adults of Elmidae (Tab. VI).

The densities of collector-gatherers were significantly different among sampling stations only during the dry period (ANOVA, $\mathrm{F}_{444}=3.05 ; \mathrm{p}=0.0277$ ), with no seasonal variation at the five stations (Tab. VI). Differences in the densities of collectors during the dry season were found only between stations 1 and 4 (Tukey's test $=0.0355$ ).

The densities of collector-filterers were significantly different among sampling stations only during the wet season (ANOVA, $\mathrm{F}_{4.44}=3.24 ; \mathrm{p}=0.0215$ ), with differences between stations 2 and 3 (Tukey's test $=0.0315$ ) and stations 2 and 5 (Tukey's test $=0.0258$ ).

Shredders showed significant variations among sampling stations during the wet season (ANOVA, $\mathrm{F}_{4,44}=$ 4.36; $p=0.0051$ ), with differences between stations 1 and 4,3 and 4 , and 4 and 5 (Tukey's test $=0.0115$ ). The densities of shredders showed a significant seasonal variation at stations 3 (ANOVA, $\mathrm{F}_{117}=4.52 ; \mathrm{p}=0.0494$ ) and 5 (ANOVA, $\left.\mathrm{F}_{1.17}=8.70 ; \mathrm{p}=0.0094\right)$

Predator densities differed among the stations only during the dry season (ANOVA, $\mathrm{F}_{444}=4.21 ; \mathrm{p}=0.0062$ ), between stations 1 and 3 (Tukey's test $=0.0196$ ), stations 1 and 4 (Tukey's test $=0.0272$ ), and stations 1 and 5 (Tukey's test $=0.0225)$. Seasonal variations in predator densities were found at station 2 (ANOVA, $\mathrm{F}_{1.17}=5.14 ; \mathrm{p}$ $=0.0376)$ and station 5 (ANOVA, $\left.\mathrm{F}_{117}=8.51 ; \mathrm{p}=0.0101\right)$.

Table II. Mean $( \pm$ SD) values of physical and chemical parameters at the Reserva Particular do Patrimônio Natural Mata Samuel de Paula Nova Lima, MG: temperature $\left({ }^{\circ} \mathrm{C}\right) ; \mathrm{pH}$; electrical conductivity $(\mu \mathrm{S} / \mathrm{cm})$; dissolved oxygen $(\mathrm{mg} / \mathrm{l})$; total alkalinity $\left(\mathrm{mEq} / \mathrm{l} \mathrm{CO}_{2}\right)$; chlorophylla $(\mu \mathrm{g} / \mathrm{l})$; total Phosphorus $(\mu \mathrm{g} / \mathrm{l})$; orthophosphate $(\mu \mathrm{g} / \mathrm{l})$; total Nitrogen $(\mu \mathrm{g} / \mathrm{l})$; nitrate $(\mu \mathrm{g} / \mathrm{l})$; nitrite $(\mu \mathrm{g} / \mathrm{l})$; ammonium ion $(\mu \mathrm{g} / \mathrm{l})$; and silicate $(\mathrm{mg} / \mathrm{l})$ in the water column; organic matter content $(\%$ D.W.) and minimum and maximum values of the sediment granulometric diversity (Shannon-Wiener H').

\begin{tabular}{|c|c|c|c|c|c|}
\hline \multirow[t]{2}{*}{ Parameters } & \multicolumn{5}{|c|}{ Sampling Stations } \\
\hline & 1 & 2 & 3 & 4 & 5 \\
\hline Temperature & $20.4 \pm 0.9$ & $20.3 \pm 0.4$ & $19.7 \pm 1.7$ & $19.97 \pm 0.6$ & $19.2 \pm 0.9$ \\
\hline $\mathrm{pH}$ & $5.20 \pm 0.60$ & $5.66 \pm 0.54$ & $6.23 \pm 1.44$ & $5.96 \pm 1.37$ & $5.71 \pm 1.32$ \\
\hline Electrical conductivity & $22.2 \pm 11.7$ & $59.8 \pm 8.09$ & $68.8 \pm 4.40$ & $52.5 \pm 5.7$ & $26.3 \pm 3.01$ \\
\hline Dissolved oxygen & $5.8 \pm 1.3$ & $4.5 \pm 1.3$ & $10.5 \pm 1.0$ & $8.7 \pm 2.6$ & $10.2 \pm 2.1$ \\
\hline Total alkalinity & $0.64 \pm 0.05$ & $0.301 \pm 0.203$ & $0.323 \pm 0.210$ & $0.371 \pm 0.184$ & $0.149 \pm 2.752$ \\
\hline Chlorophyll - a & $15.12 \pm 16.78$ & $9.83 \pm 2.81$ & $7.49 \pm 1.42$ & $10.62 \pm 4.79$ & $8.38 \pm 6.33$ \\
\hline Total Phosphorus & $19.78 \pm 13.11$ & $43.94 \pm 27.46$ & $51.39 \pm 36.02$ & $56.07 \pm 67.45$ & $32.88 \pm 30.70$ \\
\hline Orthophosphate & $4.74 \pm 1.76$ & $9.92 \pm 4.16$ & $6.73 \pm 3.90$ & $5.02 \pm 2.20$ & $6.10 \pm 4.02$ \\
\hline Total Nitrogen & $276.42 \pm 237.93$ & $213.13 \pm 129.61$ & $509.16 \pm 327.70$ & $190.61 \pm 140.63$ & $365.76 \pm 443.98$ \\
\hline Nitrate & $29.95 \pm 20.04$ & $21.69 \pm 12.41$ & $205.62 \pm 153.97$ & $25.59 \pm 13.73$ & $14.81 \pm 6.84$ \\
\hline Nitrite & $2.70 \pm 1.56$ & $2.26 \pm 1.56$ & $12.94 \pm 6.25$ & $2.56 \pm 1.86$ & $2.59 \pm 1.91$ \\
\hline Ammonium íon & $27.81 \pm 20.84$ & $13.75 \pm 12.53$ & $26.73 \pm 20.54$ & $28.49 \pm 18.21$ & $16.64 \pm 10.41$ \\
\hline Silicate & $1.40 \pm 1.43$ & $2.89 \pm 2.38$ & $3.58 \pm 1.6$ & $4.01 \pm 2.25$ & $3.23 \pm 1.74$ \\
\hline Organic matter & $32.3 \pm 22.21$ & $32.5 \pm 5.2$ & $5.6 \pm 2.1$ & $18.9 \pm 11.7$ & $6.4 \pm 2.6$ \\
\hline H' Granulometric diversity & $1.27-1.81$ & $1.38-1.72$ & $1.08-1.82$ & $0.92-1.93$ & $0.96-1.70$ \\
\hline
\end{tabular}


Table III. Taxonomic inventory and density (ind. $\mathrm{m}^{-2}$, mean $\pm \mathrm{SD}$ ) of benthic macroinvertebrates at the Reserva Particular do Patrimônio Natural Mata Samuel de Paula, Nova Lima, MG (NI, unidentified genera).

\begin{tabular}{|c|c|c|c|c|c|}
\hline \multirow[t]{2}{*}{ Taxa } & \multicolumn{5}{|c|}{ Sampling stations } \\
\hline & 1 & 2 & & 4 & 5 \\
\hline \multicolumn{6}{|l|}{ Mollusca } \\
\hline Bivalvia & $96.7 \pm 110.9$ & $422.1 \pm 647.7$ & $25.5 \pm 62.5$ & $79.2 \pm 125.3$ & $0.5 \pm 1.3$ \\
\hline \multicolumn{6}{|l|}{ Annelida } \\
\hline $\begin{array}{l}\text { Oligochaeta } \\
\text { Hirudinea }\end{array}$ & $520.1 \pm 711.2$ & $132.9 \pm 122.4$ & $47.2 \pm 70.7$ & $29.8 \pm 35.7$ & $11.4 \pm 18.5$ \\
\hline \multicolumn{6}{|l|}{ Arthropoda } \\
\hline \multicolumn{6}{|l|}{ Entognatha } \\
\hline Collembola & $1.1 \pm 2.7$ & $0.5 \pm 1.3$ & $1.1 \pm 1.7$ & $2.2 \pm 2.7$ & \\
\hline \multicolumn{6}{|l|}{ Insecta } \\
\hline \multicolumn{6}{|l|}{ Coleoptera } \\
\hline Curculionidae & $8.7 \pm 21.3$ & & & & \\
\hline \multicolumn{6}{|l|}{ Dytiscidae } \\
\hline Hydaticus sp. & $2.5 \pm 6.0$ & & & & $0.5 \pm 1.3$ \\
\hline Hydrovatus sp. & $5.5 \pm 11.9$ & & & & $0.5 \pm 1.3$ \\
\hline $\begin{array}{l}\text { Dytiscidae } \\
\text { Elmidae }\end{array}$ & $1.6 \pm 2.7$ & & & & \\
\hline Heterelmis sp. & & & $1.6 \pm 4.0$ & $18.5 \pm 37.8$ & $6.0 \pm 13.1$ \\
\hline Macrelmis sp. & & & $0.5 \pm 1.3$ & & 工 \\
\hline $\begin{array}{l}\text { Ordobrevia sp. } \\
\text { Phanocerus sp. }\end{array}$ & & & $2.1 \pm 3.9$ & $\begin{array}{l}0.5 \pm 1.3 \\
0.5+1.3\end{array}$ & $1.6 \pm 4.0$ \\
\hline Elmidae NI & $1.1 \pm 2.7$ & $6.5 \pm 15.9$ & $11.4 \pm 8.7$ & $36.9 \pm 64.6$ & $10.3 \pm 8.1$ \\
\hline Gyrinidae & & & & $0.5 \pm 1.3$ & \\
\hline Hydrophilidae & $5.5 \pm 11.9$ & & & $19.0 \pm 26.6$ & $0.5 \pm 1.3$ \\
\hline $\begin{array}{l}\text { Psephenidae } \\
\text { Psenhenus sn }\end{array}$ & & & & & \\
\hline $\begin{array}{l}\text { Psephenus sp. } \\
\text { Ptilodactylidae }\end{array}$ & & $0.5+1.3$ & $0.5+1.3$ & $6.5+9.0$ & $0.5 \pm 1.3$ \\
\hline Scirtidae & $7.4 \pm 18.1$ & $1.6 \pm 1.8$ & & $11.9 \pm 15.8$ & \\
\hline Staphylinidae & & & & $1.6 \pm 4.0$ & \\
\hline Diptera & & & & & \\
\hline Ceratopogonidae & $50.9 \pm 40.5$ & $80.3 \pm 61.4$ & $6.5 \pm 8.2$ & $12.5 \pm 12.6$ & $1.1 \pm 1.7$ \\
\hline Chaoboridae & $14.4 \pm 20.3$ & & & & \\
\hline Chironomidae & & & & & \\
\hline Chironominae & & & & & \\
\hline Apedilum sp. & & & & & $0.5 \pm 1.3$ \\
\hline $\begin{array}{l}\text { Beardius sp. } \\
\text { Chironomini NI }\end{array}$ & $252.0 \pm 365.2$ & $1.6 \pm 2.7$ & $0.5 \pm 1.3$ & $2.2 \pm 3.9$ & \\
\hline $\begin{array}{l}\text { Chironomini NI } \\
\text { Chironomus sp. }\end{array}$ & $37.6 \pm 58.7$ & $1.1 \pm 2.7$ & $87.3 \pm 214.0$ & $0.5 \pm 1.3$ & \\
\hline Cladopelma sp. & & & $2.7 \pm 4.3$ & & $1.1 \pm 2.7$ \\
\hline Cryptochironomus sp. & & $1.6 \pm 2.7$ & $2.7 \pm 6.6$ & $0.5 \pm 1.3$ & $4.3 \pm 7.6$ \\
\hline Demycriptochironomus sp. & & $1.1 \pm 2.7$ & & $0.5 \pm 1.3$ & $1.1 \pm 2.7$ \\
\hline Dicrotendipes sp. & & & $1.1 \pm 1.7$ & & \\
\hline Endotribelos sp. & $2.5 \pm 6.0$ & & & $2.7 \pm 6.6$ & \\
\hline Fissimentum sp. & $4.3 \pm 7.9$ & & & & $0.5 \pm 1.3$ \\
\hline Goeldichironomus sp. & $4.9 \pm 12.1$ & $0.5 \pm 1.3$ & $1.1 \pm 1.7$ & $0.5 \pm 1.3$ & $3.8 \pm 6.3$ \\
\hline Harnischia sp. & & $6.5 \pm 15.9$ & $1.1 \pm 2.7$ & $0.5 \pm 1.3$ & $2.2 \pm 3.9$ \\
\hline Nilothauma sp. & & $1.1 \pm 1.7$ & & & \\
\hline Parachironomus sp. & $0.5 \pm 1.3$ & $1.1 \pm 2.7$ & $7.6 \pm 7.9$ & $4.9 \pm 10.4$ & $1.6 \pm 2.7$ \\
\hline Paralauterborniella sp. & $2.7 \pm 6.6$ & & $0.5 \pm 1.3$ & & $0.5 \pm 1.3$ \\
\hline Phaenopsectra sp. & $50.5 \pm 109.9$ & $1.6 \pm 4.0$ & & & $2.2 \pm 3.4$ \\
\hline Polypedilum sp. & $7.1 \pm 6.3$ & $34.2 \pm 36.4$ & $182.8 \pm 227.4$ & $24.4 \pm 18.8$ & $38.5 \pm 88.1$ \\
\hline Stenochironomus sp. & $46.7 \pm 51.1$ & $12.5 \pm 12.8$ & & $0.5 \pm 1.3$ & $13.0 \pm 31.9$ \\
\hline Tanytarsus sp. & $499.0 \pm 754.0$ & $164.4 \pm 193.8$ & $9.8 \pm 14.3$ & $89.5 \pm 190.9$ & $6.5 \pm 9.9$ \\
\hline Tribelos sp. & $68.6 \pm 57.5$ & $3.3 \pm 6.5$ & $0.5 \pm 1.3$ & $17.9 \pm 42.3$ & $8.7 \pm 19.7$ \\
\hline Chironominae & $242.5 \pm 594.0$ & $351.6 \pm 861.1$ & & & $0.54 \pm 1.3$ \\
\hline Diptera & & & & & \\
\hline Chironomidae & & & & & \\
\hline Diamesinae & & & $1.6 \pm 4.0$ & & \\
\hline Orthocladiinae & & & & & \\
\hline Corynoneura sp. & $2.5 \pm 6.0$ & $2.7 \pm 3.8$ & $1.6 \pm 1.8$ & $2.2 \pm 3.4$ & $5.4 \pm 8.4$ \\
\hline Cricotopus sp. & $29.6 \pm 72.6$ & $2.2 \pm 5.3$ & $117.7 \pm 233.7$ & $32.0 \pm 61.9$ & $6.0 \pm 8.6$ \\
\hline Nanocladius sp. & & & $12.5 \pm 21.4$ & $3.8 \pm 4.8$ & $18.4 \pm 45.2$ \\
\hline Orthocladius sp. & $2.5 \pm 6.0$ & & $23.3 \pm 33.2$ & $4.3 \pm 7.6$ & $3.8 \pm 5.2$ \\
\hline Thienemanniella sp. & $3.3 \pm 5.0$ & $7.6 \pm 18.6$ & $15.2 \pm 18.6$ & $13.0 \pm 25.8$ & $12.5 \pm 29.0$ \\
\hline Orthocladiinae NI & $30.9 \pm 75.7$ & $52.1 \pm 127.6$ & $0.5 \pm 1.3$ & & \\
\hline Tanypodinae & & & & & \\
\hline Ablabesmyia sp. & $119.3 \pm 195.0$ & $82.5 \pm 117.9$ & $15.7 \pm 25.4$ & $31.5 \pm 70.7$ & $2.7 \pm 3.8$ \\
\hline Clinotanypus sp. & $7.4 \pm 18.1$ & & & & \\
\hline Coelotanypus sp. & & $0.5 \pm 1.3$ & & & \\
\hline Djalmabatista sp. & $14.0 \pm 18.9$ & $22.8 \pm 34.3$ & $0.5 \pm 1.3$ & $14.1 \pm 34.6$ & $2.2 \pm 3.9$ \\
\hline Labrundinia sp. & $598.2 \pm 770.0$ & $19.0 \pm 28.2$ & $2.2 \pm 3.9$ & $9.2 \pm 14.5$ & $1.6 \pm 2.7$ \\
\hline Larsia sp. & $17.3 \pm 42.3$ & $17.4 \pm 21.3$ & $3.8 \pm 7.8$ & $21.2 \pm 26.7$ & $0.5 \pm 1.3$ \\
\hline Macropelopia sp. & $2.5 \pm 6.0$ & & & & $0.5 \pm 1.3$ \\
\hline Monopelopia sp. & & & $0.5 \pm 1.3$ & & \\
\hline Procladius sp. & $4.9 \pm 12.1$ & & & & \\
\hline Tanypus sp. & $284.0 \pm 368.0$ & $132.9 \pm 196.5$ & $8.7 \pm 16.9$ & $2.2 \pm 2.7$ & $0.5 \pm 1.3$ \\
\hline $\begin{array}{l}\text { Zavrelimyia sp. } \\
\text { Tanypodinae NI }\end{array}$ & $204.0+499.7$ & $322.3+789.4$ & $\begin{array}{l}1.1 \pm 2.7 \\
0.5+1.3\end{array}$ & & \\
\hline 1апуройлае No & & & & & \\
\hline
\end{tabular}


Tab. III (cont.)

\begin{tabular}{|c|c|c|c|c|c|}
\hline Tanypodinae NI & $204.0 \pm 499.7$ & $322.3 \pm 789.4$ & $0.5 \pm 1.3$ & & \\
\hline $\begin{array}{l}\text { Culicidae } \\
\text { Dixidae }\end{array}$ & $59.7 \pm 96.8$ & $11+27$ & & $22+39$ & \\
\hline $\begin{array}{l}\text { Dixidae } \\
\text { Empididae }\end{array}$ & & $6.0+6.3$ & $5.4+6.4$ & $\begin{array}{l}2.2 \pm 5.9 \\
6.0 \pm 7.2\end{array}$ & $\begin{array}{l}1.1 \pm 1.1 \\
4.3 \pm 6.4\end{array}$ \\
\hline Psychodidae & & $1.1+1.7$ & & & \\
\hline Simuliidae & & $3.8 \pm 6.6$ & $939.1 \pm 2208.3$ & $33.6 \pm 37.7$ & $4.3 \pm 10.6$ \\
\hline Stratiomyidae & & & & $1.1 \pm 2.7$ & \\
\hline Tabanidae & & $12.5 \pm 24.5$ & & $0.5 \pm 1.3$ & \\
\hline Tipulidae & $11.9 \pm 29.2$ & $26.0 \pm 20.7$ & $4.9 \pm 12.0$ & $6.0 \pm 4.8$ & $4.3 \pm 5.3$ \\
\hline phemeroptera & & & & & \\
\hline $\begin{array}{l}\text { Baetidae } \\
\text { Baetodes sp. }\end{array}$ & & & $3.8+6.0$ & & $05+13$ \\
\hline Baetis sp. & & & $0.5 \pm 1.3$ & $0.5 \pm 1.3$ & $0.5 \pm 1.3$ \\
\hline Cloeodes sp. & $2.5 \pm 6.0$ & $0.5 \pm 1.3$ & $16.3 \pm 17.5$ & $22.2 \pm 39.5$ & $4.3 \pm 7.9$ \\
\hline Baetidae NI & $0.5 \pm 1.3$ & & & & \\
\hline \multicolumn{6}{|l|}{ Caenidae } \\
\hline Caenis sp. & & & & $1.1 \pm 2.7$ & \\
\hline Leptohyphidae & & & & & \\
\hline Traverhyphes sp. & & & & $4.3 \pm 4.9$ & $1.1 \pm 2.7$ \\
\hline Tricorythopsis sp. & & & & & $1.1 \pm 1.7$ \\
\hline \multicolumn{6}{|l|}{ Leptophlebiidae } \\
\hline $\begin{array}{l}\text { Farrodes sp. } \\
\text { Hermanella sp. }\end{array}$ & & $0.5 \pm 1.3$ & $\begin{array}{l}3.3 \pm 8.0 \\
0.5 \pm 1.3\end{array}$ & $20.6 \pm 44.3$ & $0.5 \pm 1.3$ \\
\hline Massartela sp. & & & & $0.5 \pm 1.3$ & \\
\hline Massartellopsis sp. & $4.3 \pm 7.9$ & $0.5 \pm 1.3$ & & $2.2 \pm 3.4$ & $0.5 \pm 1.3$ \\
\hline Nousia sp. & & & $0.5 \pm 1.3$ & & \\
\hline \multicolumn{6}{|l|}{$\begin{array}{l}\text { emiptera } \\
\text { eteroptera }\end{array}$} \\
\hline \multirow{2}{*}{\multicolumn{6}{|c|}{$\begin{array}{l}\text { eteroptera } \\
\text { Belostomatidae }\end{array}$}} \\
\hline Belostoma sp. & & & & & \\
\hline Gerridae & & $0.3 \pm 1.3$ & & & \\
\hline Neogerris sp. & $2.2 \pm 5.3$ & & & & \\
\hline Naucoridae & & & & $0.5 \pm 1.3$ & \\
\hline Nepidae & & & & & \\
\hline $\begin{array}{l}\text { Ranatra sp. } \\
\text { Notonectidae }\end{array}$ & & $1.1 \pm 1.7$ & & & \\
\hline $\begin{array}{l}\text { Notonectidae } \\
\text { Buenoa sp. }\end{array}$ & & & & & \\
\hline $\begin{array}{l}\text { Buenoa sp. } \\
\text { Notonecta } \mathrm{sp} .\end{array}$ & $\begin{array}{l}0.5 \pm 1.3 \\
4.1+6.5\end{array}$ & & & & $05+13$ \\
\hline \multicolumn{6}{|l|}{ Veliidae } \\
\hline $\begin{array}{l}\text { Veliidae } \\
\quad \text { Microvelia } \text { sp. }\end{array}$ & $15.8 \pm 17.5$ & $2.7 \pm 4.3$ & & $1.6 \pm 2.7$ & \\
\hline Rhagovelia sp. & $7.6 \pm 18.6$ & $2.7 \pm 3.8$ & & $1.1 \pm 2.7$ & $1.6 \pm 4.0$ \\
\hline epidoptera & $2.5 \pm 6.0$ & $0.5 \pm 1.3$ & $0.5 \pm 1.3$ & & \\
\hline Iegaloptera & & & & & \\
\hline \multicolumn{6}{|l|}{ Corydalidae } \\
\hline Corydalus sp. & & & $10.9 \pm 9.6$ & $8.1 \pm 13.8$ & \\
\hline \multirow{2}{*}{\multicolumn{6}{|c|}{ Aeshnidae }} \\
\hline & & & & & \\
\hline Aeshna sp. & $1.1 \pm 2.7$ & & & & \\
\hline Anax sp. & $2.2 \pm 3.4$ & & & & \\
\hline Gomphaeshna sp. & $0.5 \pm 1.3$ & $0.5 \pm 1.3$ & & & \\
\hline Gyanacantha sp. & $0.5 \pm 1.3$ & & & & \\
\hline Aeshnidae & $2.5 \pm 6.0$ & & & & \\
\hline Calopterygidae & & & & & \\
\hline Hetaerina sp. & & & $0.5 \pm 1.3$ & $3.8 \pm 7.8$ & $0.5 \pm 1.3$ \\
\hline Coenagrionidae & & & & & \\
\hline Acanthagrion sp. & & $0.5 \pm 1.3$ & & & \\
\hline Amphiagrion sp. & & $2.7 \pm 6.6$ & & & \\
\hline Argia sp. & $7.4 \pm 8.1$ & $4.9 \pm 4.5$ & & $2.7 \pm 3.8$ & $2.2 \pm 3.9$ \\
\hline Chromagrion sp. & $1.6 \pm 4.0$ & & & & \\
\hline Enallagma sp. & & $4.3 \pm 10.6$ & & & \\
\hline Leptobasis sp. & & $0.5 \pm 1.3$ & & & \\
\hline Nehalenia sp. & & $0.5 \pm 1.3$ & & & \\
\hline Coenagrionidae NI & $3.0 \pm 5.9$ & $6.0 \pm 8.6$ & $0.5 \pm 1.3$ & $0.5 \pm 1.3$ & $0.5 \pm 1.3$ \\
\hline Gomphidae & & & & & \\
\hline Agrigomphus sp. & & $2.7 \pm 6.6$ & & & \\
\hline Arigomphus sp. & $5.4 \pm 13.3$ & $1.6 \pm 4.0$ & & & \\
\hline Epigomphus sp. & & $1.6 \pm 2.7$ & $0.5 \pm 1.3$ & $2.7 \pm 4.3$ & \\
\hline Gomphus sp. & & $3.3 \pm 6.5$ & & & \\
\hline Hagenius sp. & & $1.6 \pm 4.0$ & & & \\
\hline Neogomphus sp./ & & & & & \\
\hline Praeviogomphus sp. & $2.5 \pm 6.0$ & $1.1 \pm 2.7$ & & $0.5 \pm 1.3$ & \\
\hline Octogomphus sp. & $76.5 \pm 125.8$ & $2.7 \pm 3.8$ & & $0.5 \pm 1.3$ & $0.5 \pm 1.3$ \\
\hline Phylogomphoides sp. & $2.5 \pm 6.0$ & & & & \\
\hline Progomphus sp. & $2.5 \pm 6.0$ & $1.1 \pm 2.7$ & & $0.5 \pm 1.3$ & \\
\hline Zonophora sp. & $2.5 \pm 6.0$ & $0.5 \pm 1.3$ & & & \\
\hline Gomphidae NI & $7.4 \pm 12.4$ & $3.8 \pm 3.2$ & & & \\
\hline Libellulidae & & & & & \\
\hline Dasythemis sp. & & $3.3 \pm 4.1$ & & & \\
\hline Erythemes sp. & & $7.1 \pm 7.0$ & & & \\
\hline Ladona sp. & & $1.1 \pm 2.7$ & & $0.5 \pm 1.3$ & $6.5 \pm 11.8$ \\
\hline Libellula sp. & $49.9 \pm 70.8$ & $15.2 \pm 18.4$ & $0.5 \pm 1.3$ & $1.1 \pm 2.7$ & $1.1 \pm 1.7$ \\
\hline
\end{tabular}




\begin{tabular}{|c|c|c|c|c|c|}
\hline Tab. III (cont.) & & & & & \\
\hline Orthemis sp. & $4.1 \pm 6.5$ & $15.2 \pm 16.8$ & $0.5 \pm 1.3$ & $0.5 \pm 1.3$ & $2.2 \pm 2.7$ \\
\hline Perithemes sp. & & & $1.6 \pm 1.8$ & $0.5 \pm 1.3$ & $10.3 \pm 22.1$ \\
\hline $\begin{array}{c}\text { Libellulidae NI } \\
\text { Meganodagrionidae }\end{array}$ & & $1.1 \pm 2.7$ & & & $1.6 \pm 4.0$ \\
\hline Megapodagrionidae & & & & & $13.0 \pm 30.3$ \\
\hline Plecoptera & & & & & \\
\hline Perlidae & & & & & \\
\hline Anacroneuria sp. & & & $6.5 \pm 15.9$ & $4.3 \pm 4.9$ & \\
\hline Enderleina sp. & & & & $2.2 \pm 5.3$ & \\
\hline Keтрпуіа sp. & & & & $2.7 \pm 6.6$ & \\
\hline Macrogynoplax sp. & & & & $8.7 \pm 15.2$ & \\
\hline Gripopterygidae & & & & & \\
\hline Paragripopteryx sp. & & & & $1.1 \pm 2.7$ & $39.6 \pm 33.7$ \\
\hline Tupiperla sp. & & & & $0.5 \pm 1.3$ & $12.5 \pm 20.3$ \\
\hline Trichoptera & & & & & \\
\hline Calamoceratidae & & & & & \\
\hline Phylloicus sp. & $2.5 \pm 6.0$ & $42.3 \pm 49.0$ & $4.9 \pm 10.4$ & $89.5 \pm 103.0$ & $8.7 \pm 18.3$ \\
\hline Glossosomatidae & & & & & \\
\hline $\begin{array}{l}\text { Protoptila sp. } \\
\text { Helicopsychidae }\end{array}$ & & & & $0.5 \pm 1.3$ & \\
\hline Helicopsyche sp. & & & & $1.6 \pm 2.7$ & \\
\hline Hydrobiosidae & & & & & \\
\hline Atopsyche sp. & & & $0.5 \pm 1.3$ & & $0.5 \pm 1.3$ \\
\hline Hydropsychidae & & & & & \\
\hline Leptonema sp. & & $0.5 \pm 1.3$ & $17.4 \pm 31.7$ & $21.7 \pm 34.4$ & $3.8 \pm 9.3$ \\
\hline Smicridea sp. & & $12.5 \pm 12.9$ & $53.7 \pm 67.7$ & $57.0 \pm 57.5$ & $32.0 \pm 41.9$ \\
\hline Hydroptilidae & & & & & \\
\hline Hydroptila sp. & $4.3 \pm 9.1$ & & $3.8 \pm 9.3$ & $0.5 \pm 1.3$ & \\
\hline Neotrichia sp. & & $0.5 \pm 1.3$ & $8.7 \pm 19.7$ & & \\
\hline Orthotrichia sp. & & & $0.5 \pm 1.3$ & & \\
\hline $\begin{array}{l}\text { Oxyethira sp. } \\
\text { Leptoceridae }\end{array}$ & $24.6 \pm 35.9$ & & & & \\
\hline Nectopsyche sp. & $7.4 \pm 18.1$ & $1.6 \pm 2.7$ & & $1.1+2.7$ & \\
\hline Oecetis sp. & $3.0 \pm 5.9$ & $4.3 \pm 5.7$ & $0.5 \pm 1.3$ & $14.1 \pm 23.0$ & $1.6 \pm 2.7$ \\
\hline Triplectides sp. & $38.9 \pm 83.0$ & $5.4 \pm 5.7$ & & $8.7 \pm 9.6$ & \\
\hline Leptoceridae NI & $0.5 \pm 1.3$ & & $0.5 \pm 1.3$ & $0.5 \pm 1.3$ & \\
\hline Odontoceridae & & & & & \\
\hline Marilia $\mathrm{sp}$ & & $6.5 \pm 6.2$ & $3.3 \pm 8.0$ & $8.7 \pm 13.3$ & \\
\hline Odontoceridae NI & & $0.5 \pm 1.3$ & & $0.5 \pm 1.3$ & \\
\hline Philopotamidae & & & & & \\
\hline Chimarra sp. & $2.5 \pm 6.0$ & $1.1 \pm 2.7$ & & $0.5 \pm 1.3$ & $0.5 \pm 1.3$ \\
\hline Wormaldia sp. & & $0.5 \pm 1.3$ & & & \\
\hline Polycentropodidae & & & & & \\
\hline Cernotina sp. & $1.1 \pm 1.7$ & $1.6 \pm 2.7$ & & & \\
\hline Cyrnellus sp. & $63.5 \pm 92.31$ & $228.4 \pm 221.5$ & & $1.1 \pm 2.7$ & $0.5 \pm 1.3$ \\
\hline Polycentropus sp. & $2.2 \pm 54.4$ & & & & \\
\hline Polycentropodidae NI & $9.9 \pm 24.2$ & $1.6 \pm 4.0$ & & & \\
\hline Hidracarina & $1.1 \pm 1.7$ & $1.1 \pm 2.7$ & $7.1 \pm 7.8$ & $0.5 \pm 1.3$ & \\
\hline Malacostraca & & & & & \\
\hline Decapoda & $297.9 \pm 426.7$ & $4.9 \pm 12.0$ & $185.5 \pm 286.6$ & & \\
\hline Platyhelminthes & & & & & \\
\hline Turbelaria & & & & & \\
\hline Planariidae & & & & $8.6 \pm 21.3$ & $0.5 \pm 1.3$ \\
\hline
\end{tabular}

Table IV. Mean $\left( \pm\right.$ SD) of the taxonomic richness values, total density (ind. $\left.\mathrm{m}^{-2}\right)$, EPT (Ephemeroptera, Plecoptera, Trichoptera), Chironomidae and Oligochaeta percentages during the rainy and dry seasons. Pielou's (J') equitability index, Shannon-Wiener (Max Min) diversity index (H'), BMWP (Biological Monitoring Work Party), ASPT (Average Score Per Taxon) and classification of the water in the studied ecosystems at the Reserva Particular do Patrimônio Natural Mata Samuel de Paula, Nova Lima, MG.

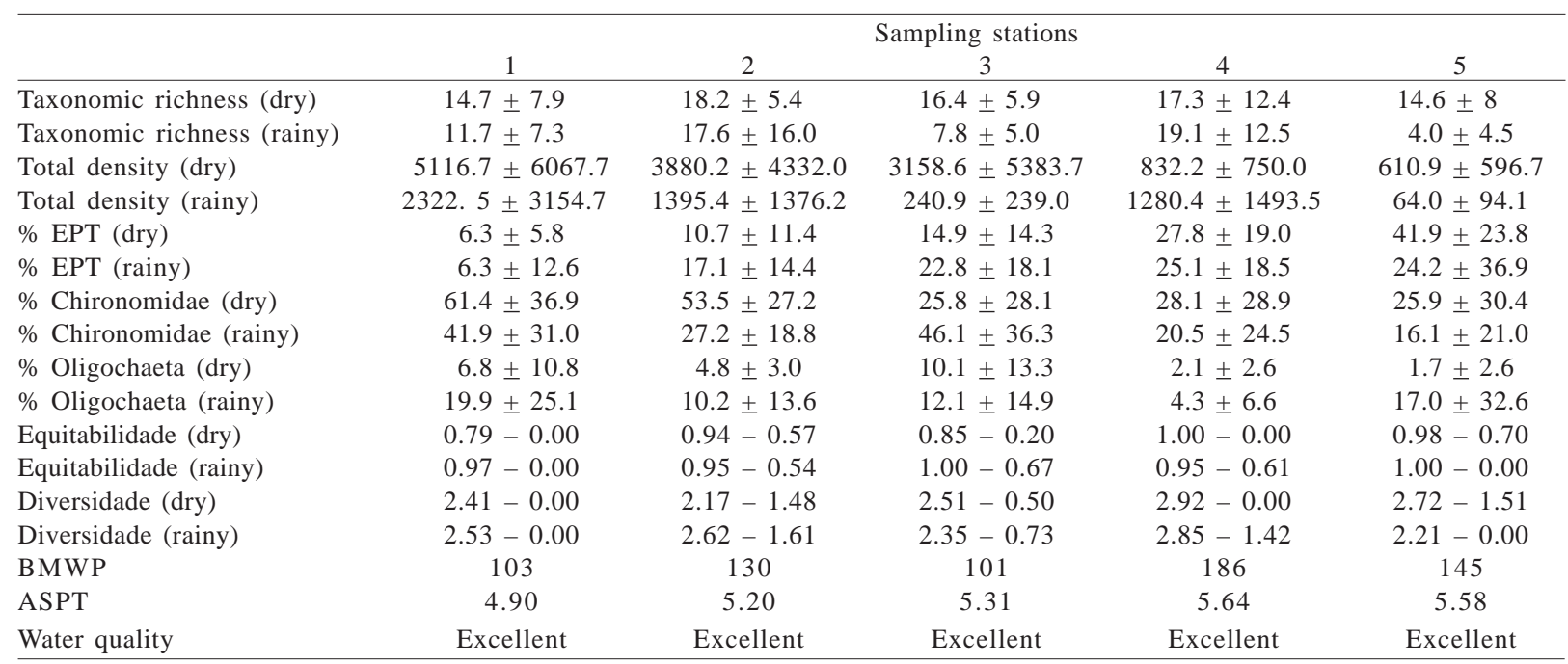


Table V. One way ANOVA $F$ values for the spatial variations (among sampled stations) and seasonal variations (between dry and wet seasons) in the taxonomic richness, total density, EPT (Ephemeroptera, Plecoptera, and Trichoptera), Chironomidae and Oligochaeta percentages in the sampling stations at the Reserva Particular do Patrimônio Natural Mata Samuel de Paula, Nova Lima, MG (*, p < $0.05 ; * *, \mathrm{p}<0.01 ; * * *, \mathrm{p}<0.001)$.

\begin{tabular}{|c|c|c|c|c|c|c|c|}
\hline \multirow{2}{*}{ Parameters } & \multicolumn{2}{|c|}{ Seasonal variation } & \multicolumn{5}{|c|}{ Spatial variation } \\
\hline & Dry & Rainy & 1 & 2 & 3 & 4 & 5 \\
\hline Richness & 0.34 & $5.32 * *$ & 0.65 & 0.03 & $11.46 * *$ & 0.09 & $11.62 * *$ \\
\hline Total density & 2.01 & 2.72 & 1.50 & 2.69 & 2.64 & 0.65 & $7.38 *$ \\
\hline$\% \mathrm{EPT}$ & $7.27 * * *$ & 1.14 & 0.00 & 1.09 & 1.07 & 0.09 & 1.46 \\
\hline$\%$ Chironomidae & 2.84 & 2.11 & 1.48 & $5.68 *$ & 1.75897 & 0.36 & 0.64 \\
\hline$\%$ Oligochaeta & 1.74 & 0.77 & 2.04 & 1.36 & 0.09 & 0.93 & 1.96 \\
\hline
\end{tabular}

Table VI. One way ANOVA $F$ values for the spatial variations (among sampled stations) and seasonal variations (between dry and wet seasons) in the functional trophic groups in the sampling stations at the Reserva Particular do Patrimônio Natural Mata Samuel de Paula, Nova Lima, MG (*, p < $0.05 ; * *, \mathrm{p}<0.01)$

\begin{tabular}{lllllccc}
\hline & \multicolumn{2}{c}{ Seasonal variation } & \multicolumn{5}{c}{ Spatial variation } \\
\cline { 2 - 7 } & Dry & Rainy & 1 & 2 & 3 & 4 & 5 \\
\hline Collector-gatherers & 3.04 & 2.13 & 0.83 & 2.74 & 1.95 & 1.70 & 2.71 \\
Collector-filterers & 1.80 & $3.24^{*}$ & 0.13 & 0.51 & 2.40 & 1.50 & 3.66 \\
Shredders & 2.37 & $4.36^{* *}$ & 1.32 & 1.76 & $4.52^{*}$ & 2.33 & $8.70^{* *}$ \\
Predators & $4.21^{* *}$ & 2.02 & 1.07 & $5.14 *$ & 0.83 & 0.53 & $8.51^{*}$ \\
Scrapers & 1.72 & 0.80 & 1.25 & 2.45 & 2.52 & 2.57 & 4.04 \\
\hline
\end{tabular}

\section{DISCUSSION}

Benthic fauna from RPPN Mata Samuel de Paula. SALLES et al. (2004) presented a list of 166 species of Ephemeroptera, distributed in 63 genera and 10 families. Most of these species were recorded for northern and southeastern Brazil. According to these authors, the most important ephemeropteran families are Baetidae and Leptophlebiidae, which together comprise $50 \%$ of the records of this order of aquatic insects. Of the 11 ephemeropteran genera in four families found in streams of the RPPN Mata Samuel de Paula, only the genera Massartelopsis Linnaeus, 1758 and Nousia Linnaeus, 1758 (Leptophlebiidae) are not found in their list. Ephemeropteran families usually are indicators of good water quality. Among the families of this order found in the area studied, the Leptophlebiidae and the Leptohyphidae are highly sensitive, while the Baetidae can tolerate some degradation of water quality due to organic input (PÉREZ, 1988; JunQueIRA \& CAMPOS, 1998).

A list of the species of Trichoptera of Brazil was proposed by PAPROCKI et al. (2004). According to these authors, in 2003 there were 378 trichopteran species. The family Hydropsychidae is the most diversified, with 107 species, and the Hydroptilidae is second with 50 species. Of the 10 families and 19 trichopteran genera found at the RPPN Mata Samuel de Paula, only two Hydropsychidae genera were recorded, while the family Hydroptilidae was represented by four genera, one of which was not recorded in the above-mentioned list (Orthotrichia Eaton, 1873). Trichopteran families are indicators of oligotrophic conditions, and usually live in fast-flowing, welloxygenated waters (PÉREZ, 1988). In this order, members of some families are highly sensitive to habitat degradation, such as Helicopsychidae and Odontoceridae, while others can survive under certain levels of pollution, such as the Hydroptilidae and
Hydropsychidae larvae (JunQueIRA \& CAMPOS, 1998). Representatives of all these were found at the RPPN Mata Samuel de Paula.

According to Tomanova et al. (2006), the collectorgatherers are the dominant trophic group in tropical streams because of the abundance of resources produced by the rapid degradation of allochthonous organic matter. In contrast, the benthic macroinvertebrate communities found at the RPPN Mata Samuel de Paula did not show a significant dominance of one trophic group, and high densities of collector-gatherers, predators, and collectorfilterers were found. According to Cummins et al. (2005), the proportions of macroinvertebrate functional feeding groups can be used as a measure of the trophic state of an aquatic ecosystem. Heterotrophic ecosystems, with low sunlight exposure and slow water flow, such as the Atlantic forest headwater streams, have as their main resource the riparian vegetation that is carried into the water by rain or wind.

Benthic macroinvertebrates and land use. The use and management of the soil and the land use in the hydrographic basin of a headwater stream has a great influence on the benthic communities (MANCINI et al., 2003). Taking into account the current state of exploitation of the region where the RPPN Mata Samuel de Paula is located, this reserve represents an important Atlantic forest remnant that preserves good ecological conditions and favors the colonization of the freshwater ecosystem by taxa that indicate optimal environmental quality, such as Gripopterygidae, Helicopsychidae, Odontoceridae, and Leptophlebiidae. At the RPPN Mata Samuel de Paula, the good ecological conditions found at the five sampling stations allowed the survival of several taxa that are not tolerant to pollution. As a consequence of the good conservation condition of these stream systems, the communities had high diversity and a high number of rare taxa, which comprised a high proportion of the 
macroinvertebrate taxonomic richness of the RPPN. The characterization of the water quality in headwater streams through indices such as BMWP and ASPT is an approach widely used by the scientific community and easily understood by environmental administrators, decision makers, and the general public (BonADA et al., 2006).

Among the most abundant taxa found in this study, most chironomid larvae are tolerant to environmental degradation and characteristic of lentic and lotic ecosystems, being able to tolerate low levels of dissolved oxygen (JUNQUEIRA \& CAMPOS, 1998). Some of these larvae are indicators of mesotrophic waters, and are usually found in sandbanks and decomposing organic matter. Simuliid larvae are characteristic of well-oxygenated running waters, and due to their filtering habit they live adhered to stable substrates such as stones and logs. Some are indicators of oligotrophic waters, and tolerate moderate levels of pollution. The Bivalvia prefer habitats where the water $\mathrm{pH}$ is neutral or over 7 and the water contains high concentrations of carbonates, which favor shell formation (PÉREZ, 1988; JUNQUEIRA \& CAMPOS, 1998). The Decapoda are shredders that have a strong influence on the trophic structure of the water bodies that they inhabit (Moulton et al., 2010).

The high number of benthic macroinvertebrate families was reflected in the high BMWP values obtained, due to the presence of intolerant taxa such as Psephenidae, Leptohyphidae, Calopterygidae, Helicopsychidae, and Odontoceridae larvae, which are all very sensitive to organic pollution (JUNQUEIRA \& CAmpos, 1998). These families receive higher scores in the BMWP and ASPT scoring procedures. Junqueira et al. (2000) classified the waters of the Cristais creek (station 3) as good quality, and assigned to this ecosystem a BMWP score of 63. These authors found pollution-sensitive taxa such as Psephenidae larvae and Gripopterygidae nymphs. In our study at the RPPN Mata Samuel de Paula, new macroinvertebrate taxa were found that were not previously recorded for the Cristais creek, increasing the previously estimated taxonomic richness of this ecosystem. This variation may be due to differences in the sampling methodologies. JUNQUEIRA \& CAmpos (1998) and JunqueIRA et al. (2000) used hand nets for sampling, whereas in this study the Surber sampler was used in triplicate, providing evidence that it may be more efficient for benthic biodiversity inventories.

Taxonomic inventories and evaluations of habitat diversity in lotic ecosystems such as those in this study are fundamental in order to use benthic macroinvertebrates as bioindicators of water quality. However, this approach must be carefully assessed, establishing reference conditions that can help to explain the variations and effects of habitat diversity on benthic macroinvertebrate diversity (BEISEL et al., 2000).

Acknowledgments. To AngloGold Ashanti Mineração and CNPq for financial support, and to CAPES for a scholarship to the first author. This study is part of the first author's master's dissertation in the Graduate Program in Ecology, Conservation and Management of Wildlife, UFMG. We thank Prof. Francisco Barbosa and his team for the physical and chemical analysis, and our colleagues of the Laboratório de Ecologia de Bentos of UFMG, especially Joana D'Arc and Pablo Moreno, for their assistance in the field, and to Raphael Ligeiro for helping with statistical analysis. The authors are grateful to the comments of two anonymous reviewers, which improved the paper. This paper was written while MC was a sabbatical visitor (CAPES fellowship No. 4959/09-4) at the IMAR, Universidade de Coimbra, Portugal.

\section{REFERENCES}

Allan, J. D. 1995. Stream Ecology: structure and function of running waters. London, Chapman \& Hall. 388p.

Armitage, P. D.; Moss, D.; Wright, J. F. \& Furse, M. T. 1983. The performance of a new biological water quality score system based on macroinvertebrates over a wide range of unpolluted running - water sites. Water Research 17:333-347.

Beisel, J. N.; Usseglio-polatera, P. \& Moreteau, J. C. 2000. The spatial heterogeneity of a river bottom: a key factor determining macroinvertebrate communities. Hydrobiologia 422/423: $163-171$.

Bonada, N.; Prat, N.; Resh, V. H. \& Statzner, B. 2006. Developments in aquatic insect biomonitoring: a comparative analysis of recent approaches. Annual Review of Entomology 51:495-523.

Callisto, M.; Ferreira, W.; Moreno, P.; Goulart, M. \& Petrucio, M. 2002. Aplicação de um protocolo de avaliação rápida da diversidade de habitats em atividades de ensino e pesquisa (MG-RJ). Acta Limnologica Brasiliensia 13(1):91-98.

Constanza, R.; D’arge, R.; Grot, R. De; Ferber, S.; Grasso, M.; Hannon, B.; Limburg, K.; Naeem, S.; O'neil, R. V.; Paruelo, J.; Raskin, R. G.; Sutton, P. \& VAn Den Bel, T. M. 1997. The values of world's ecosystem services and natural capital. Nature 387:253-260.

Cummins, K. W.; Merritt, R. W. \& Andrade, P. 2005. The use of invertebrate functional groups to characterize ecosystem attributes in selected streams and rivers in southeast Brazil. Studies on the Neotropical Fauna and Environment 40(1):69-89.

França, J. S.; Gregório, R. S.; D’Arc de Paula, J.; Gonçalves Jr, J. F.; Ferreira, F. A. \& Callisto, M. 2009. Composition and dynamics of allochthonous organic matter inputs and benthic stock in a Brazilian stream. Marine and Freshwater Research 60:990-998.

Heino, J.; Muotka, T. \& Paavola, R. 2003. Determinants of macroinvertebrate diversity in headwater streams: regional and local influences. Journal of Animal Ecology 72:425-343.

Jungueira, M. V.; Amarante, M. C.; Dias, C. F. S. \& França, E. S. 2000. Biomonitoramento da qualidade das águas da bacia do rio das Velhas (MG-Brasil) através de macroinvertebrados. Acta Limnologica Brasiliensia 12:73-87.

Junqueira, V. M. \& CAmpos, S. C. M. 1998. Adaptation of the "BMWP" method for water quality evaluation to Rio das Velhas watershed (Minas Gerais, Brazil). Acta Limnologica Brasiliensia 10(2): 125-135.

LoRENZEN, C. J. 1967. Determination of chlorophyll and pheopigments: spectrophotometric equations. Limnology and Oceanography 12:343-346.

Mackereth, F. J. H.; Heron, J. \& Talling, J. F. 1978. Water analysis: some revised methods for limnologists. Cumbria, Freshwater Biological Association. nº. 36, 120p.

MagurRan, A. E. 1991. Ecological diversity and its measurement. London, Chapman and Hall. 179p.

Mancini, L.; Formichetti, P.; Anselmo, A.; TAncioni, L.; Marchini, S. \& Sorace, A. 2003. Biological quality of running waters in protected areas: the influence of size and land use. Biodiversity and Conservation 14:351-364.

Merritt R. W. \& Cummins, K. W. 1996. An introduction to the aquatic insects of North America. Iowa, Kendall/Hunt. 750p.

Moreno, P.; França, J. S.; Ferreira, W. R.; Paz, A. D.; Monteiro, I. M. \& CAllisto, M. 2009. Use of the BEAST model for biomonitoring water quality in a neotropical basin. Hydrobiologia 630:231-242.

Moretti, M.; Loyola, R. D.; Becker, B. \& Callisto, M. 2009. Leaf abundance and phenolic concentrations codetermine the selection of case-building materials by Phylloicus sp. (Trichoptera, Calamoceratidae). Hydrobiologia 630:199-206. 
Moulton, T. P.; Magalhães-Fraga, S. A. P.; Fraga, S. A. P.; Brito, E. F. \& BARbosA, F. A. R. 2010. Macroconsumers are more important than specialist macroinvertebrate shredders in leaf processing in urban forest streams of Rio de Janeiro, Brazil. Hydrobiologia 638:55-66.

Myers, N.; Mittermeier, R. A.; Mittermeier, C. G.; Fonseca, G. A. B. \& Kent, J. 2000. Biodiversity hotspots for conservation priorities. Nature 403:853-858.

Oliveira, L. G.; Bispo, P. C.; Crisci, V. L. \& Souza, K. G. 1999. Distribuição de categorias funcionais alimentares de larvas de Trichoptera (Insecta) em uma região serrana do Brasil central. Acta Limnologica Brasiliensia 11(2): 173-183.

Paprocki, H.; Holzental, R. W. \& Blahnik, R. J. 2004. Checklist of the Trichoptera (Insecta) of Brazil. Biota Neotropica 4(1):1-22.

Paz, A.; Moreno, P.; Rocha, L. \& Callisto, M. 2008. Efetividade de áreas protegidas (APs) na conservação da qualidade das águas e biodiversidade aquática em sub-bacias de referência no rio das Velhas (MG). Neotropical Biology and Conservation 3:149-158.
Pérez, G. R. 1988. Guía para el estudio de los macroinvertebrados acuáticos del Departamento de Antioquia, Bogotá, Colciencias. 217p.

Salles, F. F.; DA-Silva, E. R.; Hubbard, M. D. \& Serrão, J. E. 2004 As espécies de Ephemeroptera (Insecta) registradas para o Brasil. Biota Neotropica 4 (2):1-34

Saunders, D. L.; Meeuwig, J. J. \& Vincent, C. J. 2002. Freshwater protected areas: strategies for conservation. Conservation Biology 16(1):30-41.

Soldner, M.; Stephen, I.; Ramos, I.; Angus, R., Wells, N. C.; Grosso, A \& CRANE, M. 2004. Relationship between macroinvertebrate fauna and environmental variables in small streams of the Dominican Republic. Water Researsh 38:863-874.

Suguio, K. 1973. Introdução à sedimentologia. São Paulo, EDUSP. $317 \mathrm{p}$.

Tomanova, S.; Goitia, E. \& Helesic, J. 2006. Trophic levels and functional feeding groups of macroinvertebrates in Neotropical streams. Hydrobiologia 556:251-264. 